\title{
RESPONSABILIDAD FINANCIERA EN LA CRISIS
}

\author{
Autores: José Luis Urquijo de la Puente \\ Exdirector del Servicio de Estudios del Banco Bilbao. \\ Laura Gismera Tierno ${ }^{1}$ \\ Profesora del Departamento de Gestión Empresarial de Universidad Pontificia \\ Comillas.
}

\section{Resumen}

En la gestación de la crisis actual concurren numerosos factores. Entre ellos están algunas actuaciones que han dañado y siguen afectando severamente los mercados de capitales. En este artículo nos referimos especialmente a tres: las limitaciones en la aplicación de fórmulas financieras, el inadecuado uso de los derivados y la ineficacia de las soluciones dadas a las crisis en los últimos 30 años. La falta de ejemplaridad queda fielmente reflejada en algunos casos como el del Banco Barings, Enron, AIG o Madoff entre otros a los que hemos hecho referencia. No sólo ha habido por lo tanto extralimitación en el uso de las formulaciones financieras, sino escándalos de fraude y falta de transparencia. Si bien es cierto que se han establecido reformas y recomendaciones por parte de las autoridades

\footnotetext{
${ }^{1}$ gismera@cee.upcomillas.es
} 
no se han llevado a cabo exhaustivamente o se han llevado con muy poca eficiencia. Es por tanto necesario pensar en un marco coherente y coordinado de regulación.

Palabras clave: Crisis financiera, formulaciones matemáticas, derivados, escándalos, reformas, recomendaciones y regulación.

\begin{abstract}
Several factors can be identified as the origin of the current economic crisis. Among them are some practices that have in the past and are still now severely damaging capital markets. In this paper we will specifically mention three: deficiencies in the application of financial formulae, improper use of derivative instruments and inadequate attempts to solve problems carried out during the last thirty years. This lack of exemplarity practices is reflected in cases like the Barings Bank, Enron. AIG or Madoff among others we will refer to. It has not only been a case of excessive and improper use of financial formulae, but also one of fraud scandals and lack of transparency. Although there has been attempts from the authorities to establish legal reforms and recommendations, little has been carried out and not always very efficiently. It is necessary to think about a consistent and coordinated regulatory framework on this matter.
\end{abstract}

Key words: financial crisis, mathematical formulae, derivatives, scandals, reforms, recommendations, regulation.

\title{
1. INTRODUCCIÓN
}

Como consecuencia de la reciente crisis económico-financiero mundial, el sistema financiero convencional ha sido puesto en entre dicho y ha sufrido muchas críticas que han dañado su imagen. A raíz de dichas críticas (algunas de las cuales se referencian en el texto), se han intentando llevar a cabo reformas para tratar de crear un sistema más transparente y que refleje con mayor precisión la economía real. A pesar de las buenas voluntades no siempre ha sido así.

En este trabajo se realiza una crítica a los modelos financieros clásicos y su aplicación en los últimos años, demostrando que nos han conducido a situaciones 
de crisis sistemáticas, de las que aún estamos luchando por salir. El objetivo del presente trabajo por lo tanto es ver dónde se ha fallado, porqué se ha fallado, en qué casos se han hecho mal o muy mal las cosas y qué reformas sería bueno que se llevaran a cabo para la transparencia, eficiencia y ética en el sistema financiero y los mercados financieros.

Al tratarse de un tema tan actual, se ha llevado a cabo una investigación exploratoria, en la que se propone alcanzar una visión general o básica del tema de estudio que abra el camino de futuras líneas de pensamiento y reflexión. Para llevar a cabo el análisis se realiza, en primer lugar, una investigación descriptiva, de las limitaciones en la aplicación de las formulaciones financieras, referenciando a la eficiencia de los mercados y a la inestabilidad de la beta de los activos. Además se describen los usos de los derivados en el mercado, señalando la inadecuación del uso de los mismos según cómo y para qué.

El trabajo consiste en la realización de una investigación deductiva; en tanto que para llevar a cabo el análisis se realizará una investigación de diferentes fuentes secundarias; a través de un enfoque cualitativo al comienzo que aporte una visión general de cómo se encuentra el mercado en la actualidad (describiendo y analizando algunos de los casos más graves de corrupción y escándalo), para pasar, con posterioridad, a un enfoque de datos cualitativos igualmente, a través de diferentes artículos y análisis de informes realizados sobre el tema que nos ocupa.

Se ha creído conveniente comentar, en primer lugar las limitaciones que presentan las principales formulaciones financieras, de amplia divulgación, y las consecuencias a las que lleva un uso inadecuado de instrumentos como los derivados financieros. Se ilustra el artículo con los ejemplos de algunos fraudes notorios, todos ellos ocurridos en este ámbito. Finalmente se concluye y comenta por dónde creen los autores deberían ir las reformas y recomendaciones para que no se vuelvan a repetir las conductas perniciosas que nos han llevado a la situación de crisis en la que todavía permanecemos.

\section{LIMITACIONES EN LA APLICACIÓN DE LAS FORMULACIONES FINANCIERAS}

Uno de los errores básicos de buena parte de los usuarios de las fórmulas ha sido el haber pensado que éstas eran infalibles sin tener en cuenta que hay estimaciones, como por ejemplo el caso de la beta, y es como la propia palabra indica: "una estimación". Buena parte de las fórmulas financieras pretenden resolver los problemas que plantean el equilibrio y riesgo en los mercados de capitales, entre otros problemas están los siguientes: 


\subsection{Eficiencia de los mercados}

Se esperaba una gran mejora logrando que los concurrentes a cada uno de los mercados estuvieran bien informados sin preferencias. Algunos creyeron que Wall Street lo había logrado en buena parte, por lo que merecía la denominación de "mercado con eficiencia fuerte". Aunque la mayoría de los autores no lo cree así, por ejemplo Malkiel considera que la eficiencia del mercado de Nueva York es semi-fuerte y como en otros mercados continúa experimentando oscilaciones originadas por informaciones privilegiadas.

\subsection{Inestabilidad de la beta de los activos}

Un medio de reducir el riesgo de una cartera de valores es diversificarlo; pero, como Markowitz demostró, dicha reducción de riesgo queda menguada en cuanto exista una correlación positiva alta entre los respectivos rendimientos de cada uno de los valores integrantes de la cartera.

Sharpe (1964) introduce una simplificación en el cálculo de Markowitz (1952) sustituyendo las múltiples covarianzas directas de los valores entre sí por las covarianzas directas con el del conjunto del mercado. Se denominó Beta a la covarianza de cada valor con el mercado.

En general, un valor con un Beta elevado es preferido por los inversores por su expectativa de beneficiarse de las subidas del conjunto de la Bolsa. El procedimiento de análisis tuvo seguidores entusiastas que creyeron resuelto el problema del riesgo de mercado. Asiduamente las principales agencias financieras en los Estados Unidos y en Londres han venido publicando las Betas de las principales acciones.

Debe advertirse que existen divergencias entre las Betas calculadas para un determinado valor, para el mismo día, según los distintos valores que se tomen como base. Y sobre todo como se ha demostrado, las Betas de un mismo valor sufren mutaciones llegando incluso a cambiar de signo.

Malkiel resume las objeciones básicas a la Beta en cuatro puntos básicos:

1. Según el índice de rendimiento del mercado que se emplee resultan diferentes betas, sin que sea posible afirmar cuál de los coeficientes hallados es el verdadero, y ni siquiera cuál es el mejor o el más útil.

2. El coeficiente Beta funciona mal a corto plazo.

3. Contra lo que preconizan sus propagadores, el coeficiente Beta de un valor varía mucho en el tiempo.

4. Se presentan serias discrepancias entre las líneas del mercado de valores (SML) determinadas teóricamente y las que reflejan la realidad del mercado. El rendimiento de los valores y de las carteras denominadas "sin riesgo" 
es mayor que el previsto teóricamente; mientras que la proporción en que aumenta el rendimiento en correspondencia con los incrementos de Beta es menor de la prevista. Pueden comprobarse diferencias importantes, incluso de signo (Urquijo 1987).

\section{INADECUADO USO DE LOS DERIVADOS}

En los últimos 60 años los mercados de capitales han aumentado sus cifras de contratación en enormes proporciones contribuyendo decisivamente a ello la elevada negociación de los derivados: opciones, futuros, swaps y otros.

Los derivados son unos instrumentos que permiten la cobertura de riesgos, y que han revolucionado el mundo financiero desde la década de los años 70. Como dice Merton Miller (1997) "estas innovaciones financieras han permitido mejorar la eficiencia en la transferencia de riesgos y han posibilitado el crecimiento del mundo financiero y económico". Estos instrumentos no son malos ni buenos en sí mismos. Todo depende del uso que se haga de ellos. Un uso fraudulento o exagerado nos conduce a situaciones como la de la crisis subprime, o la de la crisis de la deuda soberana que hemos vivido en los últimos 10 años. Premonitoria fue la frase de Warren Buffett, quien en su carta a los accionistas de Berkshire Hathaway en el año 2002, llamó a los derivados "armas de destrucción masiva, que conllevan peligros latentes que se pueden convertir en letales".

Se han dado pasos decisivos en la simplificación de la valoración de las opciones, destacando la formulación que sirve para determinar el precio de las opciones de compra y de venta debida a F. Black y M. Scholes. El trabajo de BlackScholes ha inspirado a una generación de gestores de Wall Street en los últimos cuarenta años. En febrero de 2012 el profesor británico, Ian Stewart afirmó que en los abusos cometidos con la fórmula Black-Scholes estaba la raíz de la actual crisis. Precisamente con ella las entidades emisoras de derivados han podido multiplicar su volumen de forma vertiginosa, con un aumento del riesgo que puede ser un determinante de la actual crisis. Por ejemplo la crisis de Long Term Capital Management ${ }^{2}$ a la que Black y Scholes asesoraban se produce en un entorno en el que predomina el uso de los derivados aplicados principalmente a renta fija.

A ello hay que agregar la utilización de nuevas fórmulas como la de las CDO (Colateral Debt Obligation) que ha sido el derivado preferido para colocar créditos de inferior calidad. El importe de estas operaciones ha llegado a rebasar los dos billones de dólares.

\footnotetext{
${ }^{2}$ Es un fondo de inversión libre especulativo (EEUU) que utilizó como estrategia de venta el retorno absoluto (como por ejemplo el arbitraje estadístico) combinada con un elevado apalancamiento. Este fondo quebró en 1998 y tuvo que ser rescatado por otras entidades financieras bajo la supervisión de la Reserva Federal de los Estados Unidos.
} 
Buena parte de los créditos hipotecarios "sub-prime" han tenido que ser recogidos por sus emisores, los grandes bancos de inversión, que han experimentado pérdidas que rebasan varios miles de millones de dólares (Manzano, 2008).

Este ha sido uno de los factores que más ha contribuido a la gravedad y duración de la crisis actual.

\section{FALTA DE EJEMPLARIDAD: ALGUNOS DE LOS ÚLTIMOS CASOS ESCANDALOSOS}

La falta de ejemplaridad a la que hacemos referencia se va a ilustrar con una serie de casos acontecidos. Ilustramos la falta de ejemplaridad con una serie de casos de escándalos financieros que han acontecido en los últimos años. Estos casos han sido seleccionados por su importancia y su repercusión mediática. La revisión que efectuamos no es exhaustiva, sólo pretende ser ilustrativa.

\subsection{Las actividades del Banco Barings en Singapur}

Entre los años 1992 y 1995 permitieron a Nick Leeson operar sin supervisión del Banco Barings en Londres: actuaba a la vez como jefe de operaciones de liquidación y encargado de los negocios de Barings en el Intercambio Monetario Internacional de Singapur y esto llevó a que él mismo controlara los informes que se debían presentar dentro del Banco. Leeson fue capaz de hacer pequeñas apuestas en el mercado de futuros y cubrir pérdidas al reportarlas como ganancias al Barings en Londres. Las pérdidas aumentaron cuando uno de sus colegas compró contratos cuando debería haberlos vendido. Los auditores del Barings descubrieron el fraude y una deuda de 827 millones de libras (1200 millones de dólares). Nick Leeson fue condenado por fraude y encarcelado durante seis años.

\subsection{Enron}

Era una compañía estadounidense de energía con sede en Houston y Tejas formada por la fusión de Houston Natural Gas y de InterNorth. Se trataba de la séptima empresa más grande de Estados Unidos. Su creador, Kenneth Lay; el máximo ejecutivo de la compañía, Jeffrey Skilling; y el responsable de las finanzas, Andrew Fastow; fueron los que llevaron a la empresa hacia la mayor bancarrota de EEUU hasta el momento. Jeffrey Skilling y Andrew Fastow, con

\footnotetext{
${ }^{3}$ La crisis de las hipotecas subprime es una crisis financiera, por desconfianza crediticia que se extiende inicialmente por los mercados financieros americanos y es la alarma que pone el punto de mira en las hipotecas basura europeas desde el verano del año 2006 y se evidencia al verano siguiente con una crisis bursátil.
} 
la ayuda de otros ejecutivos, escondieron miles de millones de dólares mediante manipulaciones en la contabilidad y engaños a los accionistas y a los empleados, además de tratos con los analistas de bolsa para que recomendaran sus acciones. Daban informes falsos sobre la rentabilidad de la empresa, de tal manera que las acciones iban subiendo, y la gente seguía invirtiendo. La mayoría de los empleados tenían todo su dinero en acciones de Enron que no pudieron vender. Todos los altos ejecutivos, y los que tenían información privilegiada vendieron sus acciones por un valor de mil millones de dólares; Enron quebró en tan sólo 24 días, bajando las acciones de 90 dólares a menos de un dólar y sorprendiendo a todos los EEUU y al mundo entero que pensaba que era una compañía sólida. Sin embargo, tras esa imagen de seguridad, escondía muchas pérdidas por el bajo rendimiento de las empresas de gas natural en las que había invertido por todo el mundo que costaron miles de millones, y que, mientras la compañía afirmaba su éxito, estaban acabando con ella.

\subsection{American Internacional Group (AIG).}

En el contexto de la "crisis suprime", en septiembre de 2008 American International Group (AIG) tuvo serios problemas para refinanciar una deuda de entre 10.000 y 20.000 millones de dólares. De enero a septiembre del año 2008 la empresa acumuló pérdidas por valor de 18.000 millones de dólares. Finalmente, el 17 de septiembre de 2008, la Reserva Federal de los Estados Unidos nacionalizó AIG, al borde de la quiebra, para evitar que su derrumbe arrastrara a todo el sistema financiero mundial. De este modo entregaría 85.000 millones de dólares (60.000 millones de euros) a la compañía para hacerse con el $80 \%$ de sus activos. Después del escándalo por las millonarias bonificaciones dadas posteriormente a algunos ejecutivos como bonos en AIG, 15 de 20 empleados acordaron devolverlas (24 de marzo de 2009). El fiscal general de Nueva York, Andrew Cuomo, señaló que los empleados debían devolver 50 millones de dólares. El escándalo de las bonificaciones se dio porque éstas ascendían a 165 millones de dólares, pagaderas una vez nacionalizada la empresa.

\subsection{Bernard Lawrence Madoff}

Fue el presidente de una firma de inversión que lleva su nombre y que él fundó en el año 1960. En diciembre de 2008 le detuvieron por haber estafado alrededor de 50.000 millones de dólares. Su firma de inversión, Madoff Securities, era un broker-dealer autorizado, registrado y supervisado por la SEC. La estafa se llevó a cabo con un sistema de fraude conocido como "esquema ponzi o estafa piramidal" que consiste en obtener dinero de inversores ofreciendo altas rentabilidades $(8 \%)$, pero estos intereses no son pagados con los beneficios de la empresa, sino 
con el dinero de nuevos inversores. El problema llegó cuando con la crisis muchos inversores empezaron a reclamar su dinero y era difícil captar a nuevos clientes. Al no entrar clientes nuevos, no hubo fondos con qué pagar a los antiguos y los cimientos de la pirámide se tambalearon destapando una de las mayores estafas financieras de la historia.

\section{MEDIDAS QUE URGEN}

Para afrontar la difícil situación, las autoridades financieras americanas han ido dando una serie de recomendaciones, pero ha faltado una exigencia estricta del cumplimiento de las normas (por ejemplo la Ley Sarbones-Oxley ${ }^{4}$ ); se han planificado pero no lo aplican, no lo han puesto en marcha o se ha demostrado tal laxitud en la interpretación que las ha hecho inoperantes. Las sanciones no fueron ejemplarizantes y las medidas de aplicación han sido muy complicadas, tal es así que no han dado los resultados esperados. En algunas ocasiones (como en el caso de Sarbanes-Oxley) lo que ha ocurrido es que el coste de implementación de los requerimientos exigidos por la misma a las empresas (actualización de los sistemas de información, entre otros) ha llevado a las compañías a cuestionarla y en el peor de los casos a no cumplirla.

Las crisis financieras provocan disfunciones en los mercados, y recesiones económicas más largas que otro tipo de crisis. Por este motivo, la prevención de futuras crisis financieras debe ser un objetivo para la propia industria y los órganos políticos. En el caso español, se ha implementado una seria de medidas (muchas de ellas siguiendo el Memorando de Entendimiento por el que se concedió ayuda financiera a España). La situación socioeconómica española es muy negativa, e importantes índices socioeconómicos han empeorado durante la implementación de las reformas, el propio sistema financiero español no cumple su cometido de canalizar eficientemente el ahorro. Muchas de las debilidades del sistema financiero no han sido solucionadas, por lo que siguen existiendo probabilidades de que haya futuras crisis financieras tan destructoras como la actual (Larreina, 2013). Sí es cierto que se han dado una serie de recomendaciones y medidas, propuestas como respuesta a la crisis financiera iniciada en el año 2007 en el mercado de las hipotecas subprime. Antes de enumerar recomendaciones más concretas vamos a citar las más generales: la regulación y supervisión de las entidades financieras, la regulación de los mercados financieros, la protección de consumidores e inversores, la creación de

${ }^{4}$ La Ley de Sarbanes-Oxley (Sarbanes-Oxley Act of 2002, EEUU) es también conocida como el Acta de Reforma de la Contabilidad Pública de Empresas y de Protección al Inversionista. Esta ley pretende evitar que las acciones de las empresas que cotizan en bolsa sean alteradas de manera dudosa. Su finalidad es evitar fraudes y el riesgo de bancarrota, protegiendo así al inversor. 
instrumentos más eficaces para gestionar posibles crisis financieras en el futuro y la mejora de la cooperación internacional.

Algunas recomendaciones "concretas" de las autoridades internacionales son (Uxó, 2008):

- Dictar normas más estrictas para desempeñar el cargo de broker hipotecario.

- Establecer medidas para prevenir el fraude a los consumidores que buscan una vivienda.

- El departamento del Trabajo respecto a los fondos de Pensiones y la SEC respecto a los fondos de inversiones deben vigilar que los partícipes sean informados con precisión del riesgo de las inversiones que se hagan.

- Mejorar los procedimientos de rating.

- Establecer normas claras para resolver los conflictos de intereses.

- Establecer una cámara de compensación para las operaciones "over the counter" (OTC) con derivados que permita conocer el riesgo soportado por cada partícipe, facilitando así el conocer mejor el riesgo de contrapartida que se asume en una transacción (se está haciendo en Europa y en América).

Otras reformas que urgen son las siguientes:

1. Reforma del proceso de comercialización de los créditos hipotecarios.

2. Mejora de la información a los inversores para mantener la disciplina del mercado.

3. Fortalecimiento de la gestión de riesgos en las Instituciones Financieras globales.

4. Reforzar las normas de prudencia en los mercados.

\section{CONCLUSIONES}

Al afrontar la crisis actual deben tenerse en cuenta tres amenazas aún no resueltas: a) Las valoraciones como las que han realizado las empresas de rating en los créditos hipotecarios; b) Que el mercado sigue inundado de operaciones OTC, c) Que el control que debe comenzar con el propio análisis de las fórmulas aplicadas tanto por los particulares como los bancos y las instituciones públicas que velan por los mercados se lleve a cabo; es muy importante velar por la consistencia en la aplicación de cualquier fórmula, pues los fracasos provienen de las divergencias entre lo pronosticado y lo que luego se convierte en realidad.

Hay factores negativos dominando los mercados (faltan detalles en la regulación de los derivados, las agencias de rating siguen funcionando de la misma manera, el conjunto del sistema de la valoración establecida por unos cuantos y 
la valoración sin orden). Necesitamos un consenso explícito, que tenga suficiente fuerza para poder movilizar todo el sistema. Cuando se haga una operación financiera debe hacerse de modo que se entienda e importante también es que no se utilicen lenguajes ambiguos. Algunos emisores de valores utilizan y han utilizado un lenguaje confuso para que la gente aceptara ofertas insólitas que hacen concebir esperanzas de fabulosas rentabilidades.

La innovación financiera de las últimas décadas, ha posibilitado la creación de numerosos instrumentos que han permitido tanto la transferencia del riesgo, como la transformación de las características del mismo. Así, los demandantes de financiación han podido obtenerla fácilmente acudiendo a los intermediarios tradicionales o los mercados primarios de valores puesto que, después era posible transformar las características de la financiación concedida mediante el traslado de los instrumentos de deuda a terceros que, a su vez, podían transformar esa deuda mediante su empaquetamiento, emitiendo nuevos instrumentos de deuda que tienen como colateral último los instrumentos de deuda inicialmente emitidos. Estos procesos dieron lugar a la creación de nuevos instrumentos financieros que sólo transformaban las características de unos activos pero, eso sí, creando en esta transformación nuevos riesgos.

Los volúmenes tan grandes de dinero nos han llevado al desastre. Y hemos de preguntarnos hasta qué punto algunas operaciones de matemática financiera han contribuido a la crisis actual. No se han corregido los errores (abusos de las fórmulas que no han sido detectados y, lo peor de todo, que no se han corregido). No están ni bien entendidas ni bien controladas. Estas fórmulas acentúan la crisis, pero no son los únicos protagonistas de la misma. Las fórmulas en sí no son malas, pero se han excedido en la utilización de las mismas, no se han utilizado adecuadamente. Es importante ver que la fórmula se utilice para lo que realmente es su cometido. No son ciencia exacta, su fragilidad sólo puede superarse con una crítica interna y con una constatación clara de las empresas y de la banca. Para ello se necesita seriedad por parte de las autoridades. Por ello hemos creído conveniente hablar de Beta, CAPM y Black-Scholes que han sido utilizadas abusivamente. Es un caso de extralimitación y abusos de las formulaciones financieras (además de por supuesto contemplar las limitaciones de las mismas, porque a muchos usuarios se les ha olvidado que algunas son "meras estimaciones"). También en los escándalos antes mencionados ha habido fraude y una falta de transparencia.

Autores como Torrero (2012) consideran que Keynes hubiera contemplado sin gran sorpresa el desastre financiero actual, puesto que las ideas que han sustentado el hiperdesarrollo y la internacionalización de las finanzas, han sido contrarias a las que él había sostenido en su madurez, resaltaría su desconfianza respecto a que la inversión, la variable esencial se confiara a una institución que, como aludía en la Teoría General, se asemejara a un casino. En los últimos 60 años los mercados de capitales se han hecho cada vez más complejos y su ámbito de riesgo ha crecido 
hasta la inmensidad. Sus controladores no han sido suficientemente sagaces. No han sido las nuevas fórmulas quizá es que no han sido bien digeridas aún. Los inversionistas precisan urgentemente estar protegidos por normas legales pertinentes que garanticen un control a todos los niveles para frenar las extralimitaciones, errores y abusos en la utilización de las fórmulas. La insistencia de los economistas en el desarrollo de modelos formalizados limita la percepción de los elementos que son fundamentales en el verdadero comportamiento de la realidad económica, incluyendo en ocasiones la actividad financiera (Lobejón, 2011). Ninguna alternativa aislada es perfecta y todas tienen limitaciones; sin embargo, debería pensarse en un marco coherente y coordinado de regulación que potenciara la eficacia de los mismos, especialmente en el largo plazo y en el marco de una idea clara sobre qué tipo de sistema financiero es más funcional al desarrollo.

\section{BIBLIOGRAFÍA}

AGUIRRE, Y. y MESA, R. J. (2010), "Lecciones de la crisis financiera internacional: el debate sobre la regulación financiera", Semestre económico, vol. 13, núm. 25, 61-79.

ALLAMI, C. y CIBILS, A. (2010), "Crisis financiera y regulación: propuestas heterodoxas", Política y cultura, núm. 34, 57-85.

BOOKSTABER, R. (2007), Markets, Hedge Funds, and The Perils of Financial Innovation, Hoboken, New Jersey: John Wiley \& Sons, Inc.

DOMÍNGUEZ, C. y MENÉNDEZ, J. M. (2009), "Crisis económica y financiera. La respuesta de la UE: una evaluación de las políticas aplicadas y un análisis de las ineficiencias creadas", Crisis económica y financiera: el papel de la Unión Europea, 157-185.

LARREINA, M. (2013), "La reforma del sistema financiero un año después del rescate: un éxito, pero ¿para quién?", Boletín de estudios económicos, vol. 68, núm. 209, 225-262.

LEWIS, M. (1999), "How the Eggheads Cracked", New York Times, January 24.

LOBEJÓN, L. F. (2011), "¿Qué fue de los mercados financieros eficientes? La economía, los economistas y el origen de la crisis", Revista de economía crítica, núm 11, 45-64.

MALKIEL, B. (2003), "The Efficient Market Hypothesis and its critics", Journal of Economic Perspectives, vol. 17, núm. 1, 59-82.

MANZANO, M. (2008), "La innovación financiera, la gestión del riesgo y los flujos financieros de la economía. La crisis de los préstamos subprime: crónica de una muerte anunciada", Tesis Doctoral defendida en la Universidad Complutense de Madrid. 
MARTÍNEZ, J. F. (2009), "La crisis financiera internacional y el sistema económico: los temas de fondo", Boletín económico de ICE, Información Comercial Española, núm. 2965, 23-40.

MARKOWITZ, H. (1952), "Portfolio Selection", The Journal of Finance, vol. 7, núm. 1, 77-91.

MERTON, M. (1997), "Merton Miller on Derivatives", Canadá: Ed. Wiley Investments,

MOLOGNI, C. y GISMERA, L. (2006), "El escándalo de Long Term Capital Management", Técnica Económica: Administración y Dirección de Empresas, núm. 175, 22-27.

RODIL, O. y MENEZES, F. (2012), "La crisis financiera global en perspectivas: Génesis y factores determinantes", Revista de Economía Mundial, núm. 31, 226.

SALAMA, P. (2010), "Una crisis financiera estructural", Revista de Ciencias Sociales, núm 36, 19-28.

SALAZAR, B. (2013), “¿Crisis después de la crisis?: la macroeconomía financiera después de la crisis global", Revista de economía institucional, vol. 15, núm.28, 127-158.

SHARPE, W. (1964), "Capital Asset Prices: A Theory of Market Equilibrium under conditions of risk", The Journal of Finance, vol. 19, núm.3, 425-442.

SHARPE, W. (1963), "A Simplified Model for Portfolio Analysis", Management Science, vol. 9, núm. 2, 277-293.

TORRERO, A. (2012), "Keynes y la crisis financiera actual: reflexiones a la luz de su obra". Documentos de Trabajo, núm. 8, 2012, Editorial

TREYNOR, J. (1998), "Bulls, Bears and Market Bubbles", Financial Analysis Journal, March/April , 69-74.

URQUIJO, J. L. (1987), "Tiene alguna utilidad práctica el coeficiente beta”, Asociación de licenciados en Ciencias Económicas por la Universidad Comercial de Deusto. Separata del Boletín, vol. XLII, núm 181.

UXÓ, J. (2009), "La reforma en la regulación y supervisión financiera en Estados Unidos", Boletín económico de ICE, Información Comercial Española, núm. 2976, 21-30.

\section{OTRAS PUBLICACIONES}

"Wall Street Settlement: Excerpt from Settlement with Citigroup on Salomon's Recommendations", New York Times, April 29, 2003.

"Oversight of Investment Banks' response to the Lessons of Enron", volume 1, Senate Committee on Government Affairs, December 11, 2002. 
Statement of Senator Carl Levin, Chairman, Permanent Subcommittee on Investigations, Hearing on the Role of the Financial Institutions in Enron's Collapse, July 30, 2002.

"It's Cleanup Time at Citi", New York Times, November 7, 2004.

\section{PÁGINAS WEB CONSULTADAS}

Fechas de consulta: entre febrero 2012 y octubre 2013

Stewart, I., en: www.guardian.co.uk/profile/ian-stewart-/www.lavanguardia.com www.humanite.fr/Humanite-dimanche www.elmundo.es www.forbes.com www.marketwatch.com/investing/stock/aig www.eleconomista.es www.ucm.es/info/jmas/doc/exp1.htm. www.enciclopediafinanciera.com/gestioncarteras/capm.htm. 